\section{Food and Safety}

Metabolic Aspects of Food Safety. Edited by Francis Roe. Pp. xxiii +612 . (Blackwell: Oxford and Edinburgh, 1970.) £7.

Abour ten years ago the Nuffield Foundation became concerned with the toxicological risks associated with the increase in chemical and physical treatment of food. To evaluate the hazards involved it is necessary to work with small laboratory animals, but lack of knowledge of their diseases made the investigations difficult. In 1966 the first food safety conference lead to the publication of a useful book, The Pathology of Small Laboratory Animals. In 1969 the second food safety conference considered the tests used to assess the safey of food additives and contaminants, and the problems that arise in interpretation of experimental results. It is not as easy as might be supposed to decide whether a compound is toxic to an animal even in the conditions of the experiment, without the additional problem of extrapolation to a human population and the conditions in which the compound is or could be consumed. As toxicology is studied by physiologists, biochemists and electron microscopists, there was a need for different people concerned to meet to discuss the problems. The book of the conference is disappointing, although it should be of some use to those engaged in this work.

"Metabolic Aspects" is a very misleading title, as the book is not concerned chiefly with the metabolism of food constituents, additives or contaminants, or with their effects on metabolism of the animal. "Biological Aspects" would have been more appropriate. A very heterogenous collection of twenty articles discusses basic physiology of the small intestine and of gastrointestinal absorption, renal function tests in animals and in man, and the significance of such factors as gut flora, age, sex, hormones and nutritional status of the experimental animal. The fact that protein deficiency makes animals less sensitive to compounds such as heptachlor which exert their toxic effects after metabolism, and more sensitive to compounds such as aflatoxin, the toxicity of which probably does not depend on their metabolic conversion, underlines the difficulty of applying results both to the affluent society and to the undernourished countries.

One basic problem is whether any effect produced by a chemical should be regarded as evidence of toxicity, even if it does not apparently inconvenience the animal. Thus many food additives cause an increase in liver weight, and the problem of whether this is harmful or possibly even beneficial is discussed. Obviously to assess the significance of the increase in liver weight caused by chemicals it should be compared with changes occurring naturally. However, in the present context, the long detailed discussion of the increase in liver weight occurring during pregnancy and lactation seems out of place, in the absence of sufficient comparison with chemically induced enlargement. Only a shrewd question in the discussion brought out a fundamental difference: chemicalinduced liver enlargement is frequently accompanied by hypertrophy of the smooth endoplasmic reticulum, that occurring during reproduction is not. The important question of the reversibility of natural and chemically induced changes is barely mentioned. The problem of whether an increase in weight is harmful leads to the question of what controls liver weight, and of the mechanism by which the changes are brought about. Thus it is essential "to find out not only what happened but how it happened" and to study the basic biochemical processes before the results of toxicity tests can be evaluated. Two good chapters get to grips with the problem in this way. One discusses metabolic aspects of liver tumour induction in animals by compounds known or suspected to be food contaminants, and the other discusses the ultrastructural changes.

Although apparently the aim of the book is to have a wide scope rather than to study any particular problem in depth, there are some important omissions. There is no mention of possible hazards to the brain, although the vulnerability of the central nervous system had been demonstrated spectacularly and tragically ten years before, when methyl-mercury contamination of fish was shown to be responsible for Minamata disease in Japan. More surprisingly teratogenesis is not discussed, although dyes, fungal metabolites and other possible food contaminants are known teratogens. The basic physiology, biochemistry and tests of kidney and liver function in man, are dealt with in standard textbooks. If these subjects could have been replaced by discussion more directly relevant in the present context, the book could have gone much further into the problems and would have been a more valuable contribution to the subject.

\section{M. Craddock}

\section{Tsetse and Ecology}

The Role of the Trypanosomiases in African Ecology : a Study of the Tsetse Fly Problem. By John Ford. Pp. xiv+ 568. (Clarendon: Oxford ; Oxford University: London, March 1971.) $£ 6.50$.

THE change of title of the East African Tsetse and Trypanosomiasis Research and Reclamation Organization
(EATTRRO) to East African Trypanosomiasis Research Organizatio. (EATRO) in 1956 signified a change of approach-from one in which the prime emphasis was on the insect vectors of trypanosomiasis-Glossina (tsetse flies) -to a more balanced one in which medical, veterinary, entomological and ecological aspects were to be studied as a whole. Also, the first organization was concerned with both research and reclamation, the second solely with research. John Ford served for many years with the earlier organization, only briefly with EATRO, as director, until he went to Rhodesia as director of tsetse control in 1957. He is primarily an entomologist, but a very thoughtful one, and his book is a fine attempt to put Glossina in proportion among the many other ecological factors which affect the African scene. This is a very different approach from that of the generality of British glossinologists, which was that the elimination of Glossina was a certain way of controlling trypanosomiasis; that this should be stressed to the near exclusion of all other approaches; and that economical control consistent with the poverty of the African territories affected should be able to be attained by minor interference with the Glossina vegetational environments-if only the ecology of these flies were sufficiently understood.

After extended experience of this approach in East Africa, Ford worked in Rhodesia where game elimination as a Glossina control measure had been particularly practised. He subsequently visited and studied in West Africa. He has, therefore, a unique first hand acquaintanceship with three different African regions, all sharply affected by trypanosomiasis, but in different ways. The description of trypanosomiasis situations in these three regions form the core of the book. After five introductory chapters giving essential basic information about the organisms concerned-trypanosomes, Glossina, animals and man-Ford devotes nineteen chapters to describing five sample areas, three in East Africa and one each in Rhodesia and in Nigeria. He ends with two summarizing chapters, one considering trypanosomiasis in its continental ecological aspects, the other proposing a new approach.

Basically, Ford's thesis is that, before the advent of the Europeans in Africa, the indigenous peoples had made very considerable advances towards overcoming the obstacle of trypanosomiasis by physiological and cultural adjustments in themselves and in their domestic stock and that this fact is usually overlooked. He considers the epidemics of trypanosomiasis in man and animals since the turn of the century to be direct results of the political and ecological aspects consequent on 